\title{
Warped products and the Reissner-Nordström-AdS black hole
}

\author{
Soon-Tae Hong ${ }^{1}$, Jaedong Choi $^{2}$ and Young-Jai Park ${ }^{3}$
}

\begin{abstract}
We study a multiply warped product manifold associated with the Reissner-Nordstrom-AdS metric to investigate the physical properties inside the black hole event horizons. Our results include various limiting geometries of the RN, Schwarzschild-AdS and Schwarzschild space-times, through the successive truncation procedure of parameters in the original curved space.
\end{abstract}

Keywords: warped products, Reissner-Nordstrom-AdS metric

\section{Introduction}

In order to investigate physical properties inside the black hole horizons, we briefly review a multiply warped product manifold $\left(M=B \times F_{1} \times \ldots \times F_{n}, g\right)$ which consists of the Riemannian base manifold $\left(B, g_{B}\right)$ and fibers $\left(F_{i}, g_{i}\right)$ $(i=1, \ldots, n)$ associated with the Lorentzian metric,

$$
g=\pi_{B}^{*} g_{B}+\sum_{i=1}^{n}\left(f_{i} \circ \pi_{B}\right)^{2} \pi_{i}^{*} g_{i}
$$

where $\pi_{B}, \pi_{i}$ are the natural projections of $B \times F_{1} \times \ldots \times F_{n}$ onto $B$ and $F_{i}$, respectively, and $f_{i}$ are positive warping functions. For the specific case of 
$\left(B=R, g_{B}=-d \mu^{2}\right)$, the above metric is rewritten as

$$
g=-d \mu^{2}+\sum_{i=1}^{n} f_{i}^{2} g_{i}
$$

to extend the warped product spaces to richer class of spaces involving multiple products. Moreover, the conditions of spacelike boundaries in the multiply warped product spacetimes [1] were also studied [2] and the curvature of the multiply warped product with $C^{0}$-warping functions was later investigated [3]. From a physical point of view, these warped product spacetimes are interesting since they include classical examples of spacetime such as the Robertson-Walker manifold and the intermediate zone of RN manifold $[4,5]$. Recently, the interior Schwarzschild spacetime has been represented as a multiply warped product spacetime with warping functions [3] to yield the Ricci curvature in terms of $f_{1}$ and $f_{2}$ for the multiply warped products of the form $M=R \times_{f_{1}} R \times_{f_{2}} S^{2}$. Very recently, we have studied a multiply warped product manifold associated with the BTZ (de Sitter) black holes to evaluate the Ricci curvature components inside (outside) the black hole horizons. We have also shown that all the Ricci components and the Einstein scalar curvatures are identical both in the exterior and interior of the event horizons without discontinuities for both the BTZ and dS black holes. [6]

In this paper we will further analyze the multiply warped product manifold associated with the $(3+1)$ RN-AdS metric to investigate the physical properties inside the event horizons. We will exploit the multiply warped product scheme to discuss the interior solutions in the RN-AdS black hole in section 2 and in various limiting black holes in section 3 so that we can explicitly obtain the Ricci and Einstein curvatures inside the event horizons of these metrics. 


\section{RN-ADS black hole}

In this section, to investigate a multiply warped product manifold for the Reissner-Nordström-anti-de Sitter (RN-AdS) interior solution, we start with the four-metric

$$
d s^{2}=N^{2} d t^{2}-N^{-2} d r^{2}+r^{2} d \Omega^{2}
$$

inside the horizons with the lapse function

$$
N^{2}=-1+\frac{2 m}{r}-\frac{Q^{2}}{r^{2}}-\frac{r^{2}}{l^{2}}
$$

and $d \Omega^{2}=d \theta^{2}+\sin ^{2} \theta d \phi^{2}$. Note that for the nonextremal case there exist two event horizons $r_{ \pm}(Q, l)$ satisfying the equations $0=-1+2 m / r_{ \pm}-Q^{2} / r_{ \pm}^{2}-$ $r_{ \pm}^{2} / l^{2}$. Furthermore the lapse function can be rewritten in terms of these outer and inner horizons as follows

$$
N^{2}=\frac{\left(r_{+}-r\right)\left(r-r_{-}\right)}{r^{2} l^{2}}\left(r^{2}+\left(r_{+}+r_{-}\right) r+\frac{Q^{2} l^{2}}{r_{+} r_{-}}\right)
$$

which, for the interior solution, is well defined in the region $r_{-}<r<r_{+}$. Note that the parameters $Q$ and $l$ can be rewritten in terms of $r_{ \pm}$and $m$ as follows

$$
\begin{aligned}
Q^{2} & =\frac{r_{+} r_{-}\left[2 m\left(r_{+}^{2}+r_{+} r_{-}+r_{-}^{2}\right)-r_{+} r_{-}\left(r_{+}+r_{-}\right)\right]}{\left(r_{+}+r_{-}\right)\left(r_{+}^{2}+r_{-}^{2}\right)} \\
l^{2} & =\frac{\left(r_{+}+r_{-}\right)\left(r_{+}^{2}+r_{-}^{2}\right)}{2 m-r_{+}-r_{-}}
\end{aligned}
$$

Now we define a new coordinate $\mu$ as follows

$$
d \mu^{2}=N^{-2} d r^{2}
$$

which can be integrated to yield

$$
\mu=\int_{r_{-}}^{r} \frac{d x x l}{\left[\left(r_{+}-x\right)\left(x-r_{-}\right)\left(x^{2}+\left(r_{+}+r_{-}\right) x+Q^{2} l^{2} / r_{+} r_{-}\right)\right]^{1 / 2}}=F(r) .
$$


Note that $d r / d \mu>0$ implies $F^{-1}$ is a well-defined function. Exploiting the above new coordinate (2.6), we rewrite the metric (2.1) as a warped product

$$
d s^{2}=-d \mu^{2}+f_{1}(\mu)^{2} d t^{2}+f_{2}^{2}(\mu) d \Omega^{2}
$$

where

$$
\begin{aligned}
& f_{1}(\mu)=\left(-1+\frac{2 m}{F^{-1}(\mu)}-\frac{Q^{2}}{F^{-2}(\mu)}-\frac{F^{-2}(\mu)}{l^{2}}\right)^{1 / 2}, \\
& f_{2}(\mu)=F^{-1}(\mu) .
\end{aligned}
$$

After some algebra, one can obtain the nonvanishing Ricci curvature components for the $(3+1)$ black holes with the warped product $(2.7)$ as follows

$$
\begin{aligned}
R_{\mu \mu} & =-\frac{f_{1}^{\prime \prime}}{f_{1}}-\frac{2 f_{2}^{\prime \prime}}{f_{2}}, \\
R_{t t} & =\frac{2 f_{1} f_{1}^{\prime} f_{2}^{\prime}}{f_{2}}+f_{1} f_{1}^{\prime \prime}, \\
R_{\theta \theta} & =\frac{f_{1}^{\prime} f_{2} f_{2}^{\prime}}{f_{1}}+f_{2} f_{2}^{\prime \prime}+f_{2}^{\prime 2}+1, \\
R_{\phi \phi} & =\left(\frac{f_{1}^{\prime} f_{2} f_{2}^{\prime}}{f_{1}}+f_{2} f_{2}^{\prime \prime}+f_{2}^{\prime 2}+1\right) \sin ^{2} \theta .
\end{aligned}
$$

Using the explicit expressions for $f_{1}$ and $f_{2}$ in Eq. (2.8), one can obtain identities for $f_{1}, f_{1}^{\prime}$ and $f_{1}^{\prime \prime}$ in terms of $f_{1}, f_{2}$ and their derivatives

$$
\begin{aligned}
f_{1} & =f_{2}^{\prime} \\
f_{1}^{\prime} & =-\frac{m}{f_{2}^{2}}+\frac{Q^{2}}{f_{2}^{3}}-\frac{f_{2}}{l^{2}} \\
f_{1}^{\prime \prime} & =-\frac{2 f_{1} f_{1}^{\prime}}{f_{2}}-\frac{Q^{2} f_{1}}{f_{2}^{4}}-\frac{3 f_{1}}{l^{2}}
\end{aligned}
$$

to yield the Ricci curvature components

$$
R_{\mu \mu}=\frac{Q^{2}}{f_{2}^{4}}+\frac{3}{l^{2}},
$$




$$
\begin{aligned}
R_{t t} & =-\frac{Q^{2} f_{1}^{2}}{f_{2}^{4}}-\frac{3 f_{1}^{2}}{l^{2}}, \\
R_{\theta \theta} & =\frac{Q^{2}}{f_{2}^{2}}-\frac{3 f_{2}^{2}}{l^{2}} \\
R_{\phi \phi} & =\left(\frac{Q^{2}}{f_{2}^{2}}-\frac{3 f_{2}^{2}}{l^{2}}\right) \sin ^{2} \theta,
\end{aligned}
$$

and the Einstein scalar curvature

$$
R=-\frac{12}{l^{2}} .
$$

\section{Various limiting geometries}

In this section, we analyze the various limiting geometries through the successive truncation procedure of the parameters, $Q$ and/or $l$ in the original curved RN-AdS manifold.

\subsection{Schwarzschild-AdS limit}

We first consider the RN-AdS case where the lapse function (2.2) inside the horizons is easily reduced to the Schwarzschild-AdS space, which is the limiting case of $Q \rightarrow 0$,

$$
N^{2}=-1+\frac{2 m}{r}-\frac{r^{2}}{l^{2}} .
$$

Note that the event horizon $r_{H}(l)$ satisfies the equations $0=-1+2 \mathrm{~m} / r_{H}-$ $r_{H}^{2} / l^{2}$ and the inner event horizon $r_{-}$in the RN-AdS case vanishes since $Q$ is proportional to $r_{-}$as shown in Eq. (2.4). Furthermore the lapse function (2.3) in the RN-AdS case is now reduced to the form

$$
N^{2}=\frac{\left(r_{H}-r\right)\left(r^{2} / l^{2}+r_{H} r / l^{2}+2 m / r_{H}\right)}{r},
$$

to yield the coordinate $\mu$ as follows

$$
\mu=\int_{0}^{r} \frac{d x x^{1 / 2}}{\left[\left(r_{H}-x\right)\left(x^{2} / l^{2}+r_{H} x / l^{2}+2 m / r_{H}\right)\right]^{1 / 2}}=F(r) .
$$


Moreover in our Schwarzschild-AdS limit $d r / d \mu>0$ implies $F^{-1}$ is a welldefined function so that we can rewrite the Schwarzschild-AdS metric with the lapse function (3.1) as the warped product (2.7) with

$$
\begin{aligned}
& f_{1}(\mu)=\left(-1+\frac{2 m}{F^{-1}(\mu)}-\frac{F^{-2}(\mu)}{l^{2}}\right)^{1 / 2}, \\
& f_{2}(\mu)=F^{-1}(\mu) .
\end{aligned}
$$

Using $f_{1}$ and $f_{2}$ in Eq. (3.4), we obtain the following identities

$$
\begin{aligned}
f_{1} & =f_{2}^{\prime}, \\
f_{1}^{\prime} & =-\frac{m}{f_{2}^{2}}-\frac{f_{2}}{l^{2}}, \\
f_{1}^{\prime \prime} & =-\frac{2 f_{1} f_{1}^{\prime}}{f_{2}}-\frac{3 f_{1}}{l^{2}},
\end{aligned}
$$

to yield the nonvanishing Ricci tensor components for $r<r_{H}$

$$
\begin{aligned}
R_{\mu \mu} & =\frac{3}{l^{2}} \\
R_{t t} & =-\frac{3 f_{1}^{2}}{l^{2}}, \\
R_{\theta \theta} & =-\frac{3 f_{2}^{2}}{l^{2}}, \\
R_{\phi \phi} & =-\frac{3 f_{2}^{2}}{l^{2}} \sin ^{2} \theta,
\end{aligned}
$$

and the Einstein scalar curvature

$$
R=-\frac{12}{l^{2}}
$$

which is identical to that of the RN-AdS case.

\subsection{RN limit}

We secondly consider the RN limit $[4,5]$, which is the case of $l \rightarrow \infty$ in the metric (2.1) with the lapse function for the interior solution

$$
N^{2}=-1+\frac{2 m}{r}-\frac{Q^{2}}{r^{2}} .
$$


Note that for the nonextremal case there exist two event horizons $r_{ \pm}(Q)$ satisfying the equations $0=-1+2 m / r_{ \pm}-Q^{2} / r_{ \pm}^{2}$ to yield explicit expressions

$$
r_{ \pm}=m \pm\left(m^{2}-Q^{2}\right)^{1 / 2} .
$$

Furthermore the lapse function (3.8) can be rewritten in terms of these outer and inner horizons

$$
N^{2}=\frac{\left(r_{+}-r\right)\left(r-r_{-}\right)}{r^{2}}
$$

which is well defined in the region $r_{-}<r<r_{+}$. The coordinate $\mu$ in Eq. (2.6) for the RN-AdS case is now reduced to

$$
\mu=\int_{r_{-}}^{r} \frac{d x x}{\left[\left(r_{+}-x\right)\left(x-r_{-}\right)\right]^{1 / 2}} .
$$

After some algebra, we obtain explicitly the analytic solution of Eq. (3.11) as follows

$$
\mu=2 m \cos ^{-1}\left(\frac{r_{+}-r}{r_{+}-r_{-}}\right)-\left[\left(r_{+}-r\right)\left(r-r_{-}\right)\right]^{1 / 2}=F(r),
$$

from which we have the boundary conditions

$$
\begin{aligned}
& \lim _{r \rightarrow r_{+}} F(r)=m \pi, \\
& \lim _{r \rightarrow r_{-}} F(r)=0 .
\end{aligned}
$$

Here note that $d r / d \mu>0$ implies $F^{-1}$ is a well-defined function.

Now we can rewrite the RN metric with the lapse function (3.8) as the warped product (2.7) with

$$
\begin{aligned}
& f_{1}(\mu)=\left(-1+\frac{2 m}{F^{-1}(\mu)}-\frac{Q^{2}}{F^{-2}(\mu)}\right)^{1 / 2}, \\
& f_{2}(\mu)=F^{-1}(\mu) .
\end{aligned}
$$

Exploiting $f_{1}$ and $f_{2}$ in Eq. (3.14), we obtain the following identities

$$
f_{1}=f_{2}^{\prime},
$$




$$
\begin{aligned}
f_{1}^{\prime} & =-\frac{m}{f_{2}^{2}}+\frac{Q^{2}}{f_{2}^{3}} \\
f_{1}^{\prime \prime} & =-\frac{2 f_{1} f_{1}^{\prime}}{f_{2}}-\frac{Q^{2} f_{1}}{f_{2}^{4}}
\end{aligned}
$$

to yield the nonvanishing Ricci tensor components for $r_{-}<r<r_{+}$

$$
\begin{aligned}
R_{\mu \mu} & =\frac{Q^{2}}{f_{2}^{4}}, \\
R_{t t} & =-\frac{Q^{2} f_{1}^{2}}{f_{2}^{4}}, \\
R_{\theta \theta} & =\frac{Q^{2}}{f_{2}^{2}}, \\
R_{\phi \phi} & =\frac{Q^{2}}{f_{2}^{2}} \sin ^{2} \theta .
\end{aligned}
$$

It is amusing to see that the Einstein scalar curvature (2.12) is reduced to

$$
R=0 \text {. }
$$

\subsection{Schwarzschild limit}

Thirdly, we can obtain the Schwarzschild limit without the cosmological constant from the RN lapse function (3.8) with $Q \rightarrow 0$ limit or the SchwarzschildAdS embedding of (3.1) with $l \rightarrow \infty$ limit. We obtain the vanishing Ricci tensor components and Einstein scalar curvature from (3.16) with $Q \rightarrow 0$ limit and the Einstein scalar curvature (2.12) is reduced to

$$
R=0 \text {. }
$$

which is consistent with the previous result [3].

\section{Conclusions}

We have studied a multiply warped product manifold associated with the Reissner-Nordström-anti-de Sitter (RN-AdS) metric and the limiting cases 
to evaluate the Ricci curvature components inside the charged black hole horizons. Differently from the uncharged Schwarzschild metric where both the Ricci and Einstein curvatures vanish inside the horizon, the Ricci curvature components inside the RN black hole horizons are nonvanishing, even though the Einstein scalar curvature vanishes in the interior of the charged metric. Moreover, both in the RN-AdS and in the Schwarzschild-AdS limits, both the Ricci curvature components and Einstein scalar curvatures inside the black hole horizons are nonvanishing. Here one notes that in the pure AdS and purely charged limits, the lapse functions associated with the interior four-metric are negative definite and thus one cannot apply the multiply warped products scheme to these limits. Furthermore, we have shown that all the Ricci components and the Einstein scalar curvature are identical both in the exterior and interior of the outer event horizon without discontinuities.

STH, JC and YJP would like to acknowledge financial support in part from Korea Science and Engineering Foundation Grant (R01-2001-000-000030) and the Korea Research Foundation, Grant No. KRF-2002-042-C00010, respectively.

\section{References}

[1] J.L. Flores and M. Sánchez, J. Diff. Eqn. 186, 1 (2002).

[2] S.G. Harris, Class. Quant. Grav. 17, 551 (2000).

[3] J. Choi, J. Math. Phys. 41, 8163 (2000).

[4] H. Reissner, Ann. Phys. 50, 106 (1916); G. Nordström, Proc. K. Ned. Akda. Wet 20, 1238 (1918).

[5] J. Demers, R. Lafrance, and R. C. Meyers, Phys. Rev. D 52, 2245 (1995); A. Ghosh and P. Mitra, Phys. Lett. B 357, 295 (1995); S. P. Kim, S. K. 
Kim, K. S. Soh, and J. H. Yee, Int. J. Mod. Phys. A 12, 5223 (1997);

G. Cognola and P. Lecca, Phys. Rev. D 57, 1108 (1998).

[6] S.-T. Hong, J. Choi, and Y.-J. Park, Gen. Rel. and Grav. 35 (2003).

${ }^{1}$ Department of Science Education

Ewha Womans University, Seoul 120-750, Korea

soonhong@ewha.ac.kr

${ }^{2}$ Department of Mathematics, Korea Air Force Academy

P.O. Box 335-2, Cheongwon, Chungbuk 363-849, Korea

jdong@afa.ac.kr

${ }^{3}$ Department of Physics, Sogang University, Seoul 121-742, Korea

yjpark@sogang.ac.kr 